\title{
Publishing your research study in the BMJ
}

\author{
We prioritize robust research that has the potential to impact on practice or health policy
}

\author{
José G Merino US clinical research editor
}

BMJ, Washington, DC, USA

The $B M J$ is a high impact international journal that publishes research from all specialties of medicine and is read by clinicians, researchers, and policy makers from around the world. We publish research that translates scientific discoveries into practical applications and helps doctors make better decisions in the clinic and in research, public health, and health policy settings. If your research is novel, ethical, and methodologically robust, and it deals with questions that are directly related to clinical care, public health, or healthcare policy, we invite you to submit it to the $B M J .^{1}$

To make better clinical and policy decisions, doctors and policy makers need information about risk factors for disease, the attributes of diagnostic tests, and the comparative effectiveness of different interventions (box). We value research that looks at outcomes that are relevant to patients and clinicians. We prioritize research studies that are "actionable" and may lead to changes in the way doctors advise and treat their patients. Negative studies contribute to the evidence base and are considered important if they are well designed and well executed, and if the results will help clinicians make treatment decisions. We are also interested in studies that examine robustly "why" and "how" doctors do things; those that explore how and why to offer services and specific types of care to patients; and studies that evaluate educational and quality improvement initiatives.

The $B M J$ also publishes articles that discuss research methods. These "Research Methods and Reporting" articles include manuscripts that describe innovative research and analysis methods and new ways to present research data. ${ }^{3}$ Our aim is to promote high quality clinical research. We intend our research studies to examine research methods and these articles will help researchers to design and carry out robust studies that provide good evidence. We hope, too, that they will help readers to understand research findings and editors to identify high quality studies. The $B M J$ wishes to promote transparent editorial practices and improve the process of peer review. Therefore, we also publish studies that examine the way journals, authors, and editors conduct themselves.

The $B M J$ places great emphasis on transparency because we want readers to know that the research we publish is trustworthy. ${ }^{4}$ In accordance with International Committee of
Medical Journal Editors guidance, the $B M J$ requires prospective clinical trial registration. When appraising a research paper, our editors look for evidence that the study's reported outcome measures and analyses are concordant with those stated in the study's protocol and the trial registration. ${ }^{5}$ The $B M J$ also supports the registration of protocols and results of observational studies.

To maximize usefulness and usage of data and to promote transparency, we now require that authors of clinical trials of drugs and devices commit to making their anonymised patient level data available on reasonable request. We encourage all authors of research to link the raw data from their studies to their papers. Although the BMJ has partnered with the Dryad Digital Repository to facilitate deposition and linkage of data to $B M J$ articles, authors are free to deposit their data in an institutional or other depository of their choice. ${ }^{6}$

All research published in the $B M J$ must adhere to internationally agreed ethical principles. ${ }^{7}$ We require full disclosure about authorship attribution and conflicts of interest. ${ }^{8}$ We have an open peer review system; this means that authors know who were the editors and peer reviewers involved throughout the review and editorial process. We believe this system ensures a transparent, fair, balanced, and thorough review process.

The $B M J$ offers several advantages for researchers, including high visibility for their work (the $B M J$ 's impact factor is 14.1 and 1222712 browsers from around the world access 5643 102 pages from bmj.com every month). Research published in the $B M J$ is open access - the full text of every $B M J$ research article (the definitive version published on bmj.com) is available to anyone with an internet connection, anywhere in the world, at no charge, from the day of its publication. ${ }^{9}$

Because we publish research articles under a Creative Commons license,${ }^{10}$ authors retain copyright of their work. The default license we offer is CC BY-NC but, for studies whose funders require it, we also offer a CC BY license. ${ }^{11}$ Research papers are published electronically soon after acceptance and new manuscripts are posted on bmj.com every day. The $B M J$ also automatically submits the full text of all research articles to PubMed Central, the full text repository of the US National Library of Medicine. This ensures compliance with the public access mandates of the US National Institutes of Health, the 


\section{Research that the BMJ prioritizes (not in order of importance) ${ }^{2}$}

Systematic reviews and meta-analyses of risk factors, outcomes, and treatments Studies of the risks, advantages, and properties of diagnostic tests

Clinical and population based observational studies that look at the causes, prognosis, risks, and safety of common diseases or therapies Clinical observational studies that provide support for inferences applicable to clinical practice or healthcare policy

Clinical trials that compare the effectiveness and safety of drugs, devices, or other interventions that are tested against the optimal current treatment at clinically valid doses

UK Medical Research Council, the Wellcome Trust, and other funding agencies that require public access for the research they fund.

Because medical research and clinical practice are global endeavors, we at the $B M J$ actively seek to receive submissions and to engage readers from all over the world. Several of our research editors live in Europe, two in the United States, and one in India, and our reviewers come from all continents. When making editorial decisions we consider a study's international impact. Although the $B M J$ is a single journal, and all research studies are accessible from anywhere, bmj.com offers geotargeted content for UK, US, Indian, and international readers.

If you are engaged in research that may change the way we practice medicine, set healthcare priorities, or conduct and disseminate research, we are interested in your work and we encourage you to submit it to the $B M J$.

Competing interests: I have read and understood the BMJ Group policy on declaration of interests and declare the following interests: I am employed by and perform clinical work for Johns Hopkins Community Physicians.

Provenance and peer review: Commissioned; not externally peer reviewed.

1 BMJ. Resources for authors. www.bmj.com/about-bmj/resources-authors.

2 BMJ. Is the BMJ the right journal for my research article? www.bmj.com/about-bmj/ resources-authors/forms-policies-and-checklists/bmj-right-journal-my-research-article.

3 BMJ. Research methods and reporting. www.bmj.com/about-bmj/resources-authors/ article-types/research-methods-and-reporting.

4 BMJ. Transparency policy. www.bmj.com/about-bmj/resources-authors/forms-policiesand-checklists/transparency-policy.

5 BMJ. Research. www.bmj.com/about-bmj/resources-authors/article-types/research.

6 Dryad. www.datadryad.org/discover?field=prism. publicationName filter\&fq=location:I2\& fq=prism.publicationName_filter\%3Abmj\%5C\%7C\%5C\%7C\%5C\%7CBMJ.

7 BMJ. Ethics approval of research. www.bmj.com/about-bmj/resources-authors/formspolicies-and-checklists/ethics-approval-research.

8 BMJ. Authorship and contributorship. www.bmj.com/about-bmj/resources-authors/articlesubmission/authorship-contributorship.

9 BMJ. Copyright, open access, and permission to reuse. www.bmj.com/about-bmj/ resources-authors/forms-policies-and-checklists/copyright-open-access-and-permissionresources
reuse.
Creative

10 Creative Commons. http://creativecommons.org/.

11 Creative Commons. About the licenses. http://creativecommons.org/licenses/

Cite this as: BMJ 2013;346:12433

๑) BMJ Publishing Group Ltd 2013 